\title{
SPECTRAL LOCALIZATION ANALYSIS IN NONLOCAL SOFTENING MATERIALS
}

\author{
GIOVANNI DI LUZIO* \\ *Politecnico di Milano \\ Milan, Italy \\ e-mail: giovanni.diluzio@polimi.it
}

Key words: Softening, Plasticity, Damage Mechanics, Localization, Nonlocal Models, Gradient Models, Spectral Analysis, Harmonic Waves.

\begin{abstract}
The paper shows that spectral wave propagation analysis reveals in a simple and clear manner the effectiveness of various regularization techniques for softening materials, such as plasticity and damage models. It is verified the effectiveness of the nonlocal integral-type approach with an unconventional nonlocal formulation introduced in 1994 by Vermeer and Brinkgreve, which was called "over-nonlocal" by Di Luzio and Bažant in 2005 because it uses a linear combination of local and nonlocal variables in which a negative weight imposed on the local variable is compensated by assigning to the nonlocal variable weight greater than 1 . The spectral approach readily confirms that the nonlocal integral-type generalization of softening plasticity with an additive format gives correct localization properties only if an over-nonlocal formulation is adopted. By contrast, the nonlocal integral-type generalization of softening plasticity with a multiplicative format provides realistic localization behavior, just like the nonlocal integral-type damage model, and thus does not necessitate an over-nonlocal formulation. The localization behavior of explicit and implicit gradient-type models is also analyzed demonstrating that they have very different localization properties.
\end{abstract}

\section{INTRODUCTION}

Already in the mid 1970s it was recognized that a continuum formulation for softening materials leads to serious problems: the boundary value problem becomes ill-posed, and the numerical calculations cease to be objective, exhibiting pathological spurious mesh sensitivity, incorrect size effect, and excessive damage localization as the mesh is refined. To recover a well-posed problem and to prevent the damage from localizing into a zone of zero volume, the continuum theory must be complemented by certain conditions called localization limiters, involving a characteristic length of the material [1-3].

A broad class of localization limiters is based on the concept of a nonlocal continuum. The nonlocal concept was introduced in the $1960 \mathrm{~s}$ [4,5] for elastic deformations and in the 1980s expanded to hardening plasticity. A nonlocal continuum is a continuum in which the stress at a point depends not only on the strain at that point but also on the strain field in the neighborhood of that point. Bažant et al. [6] introduced the nonlocal concept as a localization limiter for a strain-softening material. This formulation was later improved in the form of the nonlocal damage theory [7]. To improve the performance in large post-peak deformations, Vermeer and Brinkgreve [8] proposed a novel formulation in which a nonlocal averaging integral with a weight function everywhere positive is modified by multiplying the weight function by a factor greater than 1 and compensating 
for it by adding at the center a negative deltafunction spike, equivalent to subtracting the local variable (this is unlike applying the common rule of mixture because the nonlocal component in the binary mixture of the local and nonlocal variables has a weight greater than 1 , compensated by a negative weight for the other component). This formulation was termed overnonlocal in [14]. Another well-known regularizing technique is based on an explicit secondorder gradient model, which is weakly nonlocal [3, 9-12]. More recently, Peerlings et al. [13] developed an effective (strongly nonlocal) modification of the gradient approach in which the nonlocal strain is solved from a Helmholtz differential equation.

In this paper some of the main results presented in [14] are reported. Firstly, the basic concept of nonlocal formulation of integraltype is presented with the relation between it and the gradient enhancements. Secondly, the over-nonlocal model of integral-type will be applied to nonlocal plasticity and compared with the explicit and implicit gradient enhancement techniques. Thirdly, the over-nonlocal model of integral-type will be applied to nonlocal damage model and compared with the explicit and implicit gradient enhancement techniques. Dispersion analysis will show that the nonlocal integral-type generalization of classical softening plasticity model with an additive format of the softening law exhibits realistic localization properties only if an over-nonlocal formulation is considered. By contrast, the nonlocal integral-type generalization of softening plasticity with a softening hardening law written in the multiplicative format (ductile damage model [15, 16]) provides realistic localization behavior, just like the nonlocal integraltype damage model, and thus does not necessitate an over-nonlocal formulation. The approach followed in this study has been pursued in several previous studies: for a gradient plasticity model, wave propagation and dispersion were investigated in [17, 18]; for the damage model regularized by a nonlocal formulation or by gradient-type enhancement, a dispersion analysis was conducted in [19-22]. Other important studies on the nonlocal plasticity model have been presented in [23, 24].

\section{BASIC CONCEPT OF NONLOCAL IN- TEGRAL AND GRADIENT FORMU- LATIONS}

The integral nonlocal model, in general, consists in replacing a certain local variable $f(\mathbf{x})$, characterizing the softening damage of material, by its nonlocal counterpart $\bar{f}(\mathbf{x})$. The nonlocal variable is defined as

$$
\bar{f}(\mathbf{x})=\int_{V} \alpha^{*}(\mathbf{x}, \boldsymbol{\xi}) f(\boldsymbol{\xi}) \mathrm{d} V(\boldsymbol{\xi})
$$

where $V$ is the volume of the structure, $\mathbf{x}, \boldsymbol{\xi}$ are the coordinates vectors, and $\alpha^{*}(\mathbf{x}, \boldsymbol{\xi})$ is the normalized nonlocal weight function defined as

$$
\alpha^{*}(\mathbf{x}, \boldsymbol{\xi})=\frac{\alpha(\boldsymbol{\xi}-\mathbf{x})}{\int_{V} \alpha(\boldsymbol{\xi}-\mathbf{x}) \mathrm{d} V(\boldsymbol{\xi})}
$$

where $\alpha(\mathbf{x}-\boldsymbol{\xi})$ is the basic nonlocal weight function which is often taken as a bell-shaped function or the Gauss' error function and $\int_{V} \alpha(\mathbf{x}-\boldsymbol{\xi}) \mathrm{d} V(\boldsymbol{\xi})$ is a constant if the unrestricted averaging domain does not tend to protrude outside the boundaries.

Originally Vermeer and Brinkgreve [8] introduced a refinement of the standard nonlocal formulation in the form of an over-nonlocal formulation in which the local and the nonlocal variables are linearly combined as follows:

$$
\hat{f}(\mathbf{x})=m \bar{f}(\mathbf{x})+(1-m) f(\mathbf{x})
$$

here $\hat{f}(\mathbf{x})$ is the over-nonlocal average of the variable $f(\mathbf{x}), \bar{f}(\mathbf{x})$ is the nonlocal variable obtained from Eq. 11, and $m$ is an empirical coefficient (over-nonlocal parameter) greater than 1 has showed by many authors [14].

The refinement of Eq. 3 can also be obtained by rewriting the normalized nonlocal weight function, Eq. 2, in the following way:

$$
\begin{aligned}
\alpha_{m}^{*}(\mathbf{x}, \xi)= & (1-m) \delta(\boldsymbol{\xi}-\mathbf{x}) \\
& +m \frac{\alpha(\boldsymbol{\xi}-\mathbf{x})}{\int_{V} \alpha(\boldsymbol{\xi}-\mathbf{x}) \mathrm{d} V(\boldsymbol{\xi})}
\end{aligned}
$$

where $\delta$ denotes the Dirac delta function; $m$ is here called the over-nonlocal parameter, because $m>1$ assigns to the nonlocal averaging 
integral in the foregoing equation excess weight compared to the standard nonlocal formulation, in which $m=1$. Thus, the over-nonlocal operator can be defined as

$$
\hat{f}(\mathbf{x})=\int_{V} \alpha_{m}^{*}(\mathbf{x}, \boldsymbol{\xi}) f(\boldsymbol{\xi}) \mathrm{d} V(\boldsymbol{\xi})
$$

in which the subscript of $\alpha$ indicates that averaging operator depends on $m$.

The gradient-type models can be considered as differential approximation of the integraltype nonlocal models. This correspondence helps to explain the role of the over-nonlocal parameter. Let the local variable $f$ be expanded around $x$ into the Taylor series:

$$
\begin{array}{r}
f(z)=f(x)+f_{, x}(z-x)+\frac{1}{2} f_{, x x}(z-x)^{2} \\
+o\left[(z-x)^{2}\right]
\end{array}
$$

Averaging the variable $f(z)$ through Eq. 5 and neglecting the terms of third and higher order in terms of the distance $|z-x|$, we obtain:

$$
\begin{aligned}
\hat{f}(x) \simeq & f(x) \int_{L} \alpha_{m}^{*}(y-x) \mathrm{d} y+ \\
& f_{, x} \int_{L}(y-x) \alpha_{m}^{*}(y-x) \mathrm{d} y+ \\
& \frac{1}{2} f_{, x x} \int_{L}(y-x)^{2} \alpha_{m}^{*}(y-x) \mathrm{d} y
\end{aligned}
$$

The weight function $\alpha(x-y)$ is normalized such that $\alpha(0)=1$ and $\int_{L} \alpha(z) \mathrm{d} z=\ell$. Moreover, in an infinite specimen (or at points sufficiently far from the boundary of a finite specimen), the function $\alpha(x-y)$ depends only on the distance $|z-x|$. In Eq. 7, due to symmetry the term $\int_{L}(y-x) \alpha(x-y) \mathrm{d} y$ vanishes and the term $\int_{L}(y-x)^{2} \alpha(x-y) \mathrm{d} y$ is equal to $\ell^{2}$. Consequently, the expression in Eq. 7 may be written as

$$
\begin{aligned}
\hat{f}(x)=f(x)+m \frac{\ell^{2}}{2} f(x)_{, x x}= & \\
& f(x)+\frac{\ell_{o}^{2}}{2} f(x)_{, x x}
\end{aligned}
$$

The gradient-type models, as showed, approximate the nonlocal integral-type models. However, there exist two different gradient-type models: the explicit and the implicit gradient enhancement. The explicit gradient models are those directly obtained by simply introducing in the constitutive law certain gradient terms. They are weakly nonlocal, because only the infinitely close neighborhood of a point directly affects the response. The implicit gradient models are those in which the constitutive law is made to depend on a nonlocal field that is obtained as the solution of a separate differential equation (Helmholtz equation). The implicit models are strongly nonlocal, just like the integral-type models, because a non-vanishing neighborhood of a point directly affects the solution.

\section{LOCALIZATION ANALYSIS OF NON- LOCAL PLASTICITY}

Consider now a simple plasticity model in the one-dimensional case. Local plasticity with linear hardening is described by the equations

$$
\begin{gathered}
\sigma=E\left(\epsilon-\epsilon_{p}\right) \\
f\left(\sigma, \sigma_{Y}\right)=|\sigma|-\sigma_{Y} \text { and } \sigma_{Y}=\sigma_{0}+H \kappa
\end{gathered}
$$

The evolution law of the plastic strain is written as

$$
\begin{gathered}
\dot{\epsilon}_{p}=\dot{\kappa} \frac{\partial f}{\partial \sigma}=\dot{\kappa} \operatorname{sgn}(\sigma) \\
\dot{\kappa} \geq 0 \quad f\left(\sigma, \sigma_{Y}\right) \leq 0 \quad \dot{\kappa} f\left(\sigma, \sigma_{Y}\right)=0
\end{gathered}
$$

where $\sigma$ is the stress (considered to be positive, i.e. tensile), $\epsilon$ is the strain, $E_{0}$ is Young's modulus, $\epsilon_{p}$ is the plastic strain, $\sigma_{Y}$ is the current yield stress, $H$ is the plastic modulus (for strainsoftening considered as negative), $\sigma_{0}$ is the initial yield limit, and $\kappa$ is the plastic softening parameter, taken to coincide with the cumulative plastic strain, i.e. $\dot{\kappa}=\left|\dot{\epsilon}_{p}\right|$.

Let us consider the one-dimensional equation of motion linearized around an initial strained homogeneous state, in which $\epsilon(x)=\epsilon_{0}=$ constant, and $\hat{\kappa}(x)=\kappa(x)=$ $\kappa_{0}=$ constant,

$$
\dot{\sigma}_{, x}=\rho \dot{u}_{, t t}
$$

where $\dot{u}$ is the time rate of the incremental displacement $u$ perturbing the initial state, and $\rho$ 
is the mass density of the bar. The relation between the rate of deformation and the time derivative of the incremental displacement rate is

$$
\dot{\epsilon}=\dot{u}_{, x}
$$

We assume that all the material is in a softening state (this is a classical assumption in the analysis of localization). Its non-trivial equilibrium solution (different from the initial homogeneous state) yields bifurcation points in statics. Starting from a homogeneous state of the solid, assumed to be so large that the boundary effect is negligible, we consider a harmonic wave solutions of frequency $\omega$ and wave number $k$ propagating through a very long bar with the following form

$$
\begin{aligned}
& \dot{u}(x, t)=\dot{u}_{0} e^{i(k x-\omega t)} \\
& \dot{\kappa}(x, t)=\dot{\kappa}_{0} e^{i(k x-\omega t)}
\end{aligned}
$$

where $i=$ imaginary unit. The over-nonlocal average of the plastic variable and its time derivative are

$$
\begin{aligned}
& \hat{\kappa}(x)=\int_{L} \alpha_{m}^{*}(\xi-x) \kappa(\xi) \mathrm{d} \xi \\
& \dot{\hat{\kappa}}(x)=\int_{L} \alpha_{m}^{*}(\xi-x) \dot{\kappa}(\xi) \mathrm{d} \xi
\end{aligned}
$$

where $\alpha_{m}^{*}(\xi-x)=(1-m) \delta(\xi-x)+m \alpha^{*}(\xi-x)$ and $\delta(\xi-x)$ is the Dirac delta function.

\subsection{Plasticity with nonlocal plastic strain}

Let us consider a nonlocal formulation of softening plasticity in which the plastic strain in the stress-strain law (9) is replaced by its nonlocal average

$$
\begin{gathered}
\sigma=E\left(\epsilon-\hat{\epsilon}_{p}\right) \quad \text { and } \\
f\left(\sigma, \sigma_{Y}\right)=E\left(\epsilon-\hat{\epsilon}_{p}\right)-\left(\sigma_{0}+H \kappa\right)
\end{gathered}
$$

Remembering that $\dot{\epsilon}=\dot{u}_{, x}$, differentiating Eq. 18 with respect to time and spatial coordinate $x$, and using the linearized equation of motion (Eq. 13), we obtain

$$
E\left(\dot{u}_{, x x}-\dot{\hat{\kappa}}_{, x}\right)=\rho \dot{u}_{, t t}
$$

Starting from an initial uniform plastic strain state, the loading-unloading condition (Eq. 10) requires that

$$
\dot{f}=E \dot{u}_{, x}-E \dot{\hat{\kappa}}-H \dot{\kappa}=0
$$

Substituting the harmonic wave solutions in Eq. 15 and their partial derivatives with respect to $x$ and $t$ into Eqs. 19 and 20, we obtain a system of two equations that must be verified at every point $x$ and for every instant $t$. Since this is a homogeneous linear system of two equations for $\dot{u}_{0}$ and $\dot{\kappa}_{0}$, the solution differs from the trivial one only if the determinant of the coefficient matrix is equal to zero, i.e.,

$$
\begin{aligned}
-E H k^{2}+\rho \omega^{2}[H+E(1-m+ & \\
\left.\left.\frac{m}{\ell} A(k)\right)\right] & =0
\end{aligned}
$$

where

$$
A(k)=\int_{L} \alpha(z) e^{i k z} \mathrm{~d} z
$$

For an infinite bar $(L \rightarrow \infty), A(k)$ is the Fourier transform of the weight function $\alpha(z)$. The Eq. 21 implies that no wave number $k$ makes the phase velocity to vanish, i.e. $c_{p}=\omega / k=$ 0 . Consequently the harmonic waves are nondissipative and the constitutive model is unable to reproduce the localization band due to the softening. Therefore, for each value of the parameter $m$ the plasticity model with the average of the plastic strain is not able to restore the well-posedness of the problem when the softening occurs.

Considering an explicit gradient enhancement, which is obtained by replacing the nonlocal variable with its approximation in Eq. 8 , the Eq. 17 is now written as

$$
\dot{\hat{\kappa}}=\dot{\kappa}+\frac{\ell^{2}}{2} \dot{\kappa}_{, x x}
$$

The explicit gradient formulation of the plasticity model in Eq. 18 is obtained by replacing the plastic strain in the stress-strain law with the sum of the variable and its second derivative. Assuming that all the material is initially in a softening homogeneous state, one gets from 
Eqs. 19 and 20, together with Eq. 23, the following equation system

$$
\begin{gathered}
E\left(\dot{u}_{, x x}-\dot{\kappa}_{, x}-\frac{\ell^{2}}{2} \dot{\kappa}_{, x x x}\right)=\rho \dot{u}_{, t t} \\
\dot{f}=E \dot{u}_{, x}-E\left(\dot{\kappa}+\frac{\ell^{2}}{2} \dot{\kappa}_{, x x}\right)-H \dot{\kappa}=0
\end{gathered}
$$

Consider harmonic waves of frequency $\omega$ and wave number $k$, propagating along the bar with the velocity and cumulative plastic strain field given by Eq. 15. Substituting Eq. 15 and its partial derivatives with respect to $x$ and $t$ into Eqs. 24 and 40, we obtain a system of two equation that must be verified at every point $x$ and for every instant $t$. Because this is a homogeneous system of two linear equations, a nontrivial solution exists if and only if the determinant of the coefficient matrix vanishes;

$$
\begin{array}{r}
\left(E k^{2}-\rho \omega^{2}\right)\left[\left(1-\frac{\ell^{2}}{2} k^{2}\right) E+H\right]- \\
E^{2} k^{2}\left(1-\frac{\ell^{2}}{2} k^{2}\right)=0
\end{array}
$$

In statics $(\omega=0)$, we have

$$
H E k^{2}=0
$$

The explicit gradient enhancement of the plasticity model gives harmonic waves that are nondissipative, and consequently, is unable to reproduce the localization band due to the softening.

Another important class of gradient-type models consists of the implicit gradient-type models, in which Eq. 23 is rearranged as follows

$$
\dot{\hat{\kappa}}-\frac{\ell^{2}}{2} \dot{\hat{\kappa}}_{, x x}=\dot{\kappa}
$$

which is the Helmholtz equation. The implicit gradient formulation of the plasticity model in Eq. 18 is obtained by replacing the plastic strain in the stress-strain law with the nonlocal variable obtained by the solution of Eq. 28. Two differentiations of Eq. 20 with respect to $x$, together with Eq. 28, give

$$
\dot{\hat{\kappa}}=\dot{\kappa}+\frac{\ell^{2}}{2 E}\left(E \dot{u}_{, x x x}-H \dot{\kappa}_{, x x}\right)
$$

Rearranging Eqs. 19, 20 and 29 and substituting the harmonic waves of frequency $\omega$ and wave number $k$ from Eq. 15 and its partial derivatives respect to $x$ and $t$ one gets a system of two homogeneous linear equations, whose non-trivial solution exists if and only if the determinant of the coefficient matrix vanishes, i.e.

$$
\begin{gathered}
\left(E k^{2}+E \frac{\ell^{2}}{2} k^{4}-\rho \omega^{2}\right) \\
\left(E+H+H \frac{\ell^{2}}{2} k^{2}\right)-E k^{2} \\
\left(E+H \frac{\ell^{2}}{2} k^{2}\right)\left(1+\frac{\ell^{2}}{2} k^{2}\right)=0
\end{gathered}
$$

For statics $(\omega=0)$, Eq. 30 becomes

$$
E k^{2} H\left(1+\frac{\ell^{2}}{2} k^{2}\right)=0
$$

This equation implies that there exists no nonzero critical wave number. This means that the waves are non-dissipative. This is the same behavior as observed for the nonlocal (Eq. 21) and the explicit gradient-type (Eq. 27) formulation of this plasticity model.

\subsection{Plasticity with nonlocal plastic variable}

Consider now a simple over-nonlocal integral-type plasticity model, in which the plastic variable is replaced by its over-nonlocal counterpart in the current yield stress. The constitutive relations are given by

$$
\sigma=E\left(\epsilon-\epsilon_{p}\right) \quad \text { and } \quad \sigma_{Y}=\sigma_{0}+H \hat{\kappa}
$$

Differentiating Eq. 9 with respect to time and spatial coordinate $x$ and using the linearized equation of motion (Eq. 13, we obtain

$$
E\left(\dot{u}_{, x x}-\dot{\kappa}_{, x}\right)=\rho \dot{u}_{, t t}
$$

Starting from an initial uniform plastic strain state, the loading-unloading condition (Eq. 10) requires that

$$
\dot{f}=E \dot{u}_{, x}-E \dot{\kappa}-H \dot{\hat{\kappa}}=0
$$

Substituting the harmonic wave solutions of Eq. 15 and their partial derivatives with respect to $x$ 
and $t$ into Eqs. 33 and 34 , one obtains a homogeneous linear system of two equations for $\dot{u}_{0}$ and $\dot{\kappa}_{0}$, and its solution differs from the trivial one only if the determinant of the coefficient matrix is equal to zero, i.e.,

$$
\begin{array}{r}
-\left[E+H\left(1-m+\frac{m}{\ell} A(k)\right)\right] \\
\left(E k^{2}-\rho \omega^{2}\right)+E^{2} k^{2}=0
\end{array}
$$

For statics $(\omega \rightarrow 0)$, Eq. 35 becomes

$$
\left[1-m+\frac{m}{\ell} A(k)\right] E H k^{2}=0
$$

The harmonic wave perturbations about the initial state, Eq. 15, do not remain homogeneous if Eq. 36 is satisfied. Thus we look for bifurcation points. Let us consider the Gauss weight function $\alpha(z)=\exp \left(-\pi z^{2} / \ell^{2}\right)$, such that $\alpha(0)=1$ and $\int_{-\infty}^{+\infty} \alpha(z) \mathrm{d} z=\ell$. Noting that $\int_{-\infty}^{+\infty} \exp \left(-z^{2}\right) \mathrm{d} z=\sqrt{\pi}$, Eq. 22 gives the Fourier transform $A(k)=\ell e^{-k^{2} \ell^{2} / 4 \pi}$. Substituting this value of $A(k)$ into Eq. 36, we search for the value of $k=k_{c r}$ for which Eq. 36 can be satisfied for $k \neq 0$;

$$
k_{\mathrm{cr}}=\frac{2 \sqrt{\pi}}{\ell} \sqrt{\ln \left(\frac{m}{m-1}\right)}
$$

The corresponding critical wavelength is

$$
\lambda_{\mathrm{cr}}=2 \pi / k_{\mathrm{cr}}=\ell \sqrt{\pi}\left[\ln \left(\frac{m}{m-1}\right)\right]^{-1 / 2}
$$

Note that for $m \rightarrow 0$, or for $\ell \rightarrow 0$ (local strain-softening model), the critical wavelength $\lambda_{c r}$ tends to zero, i.e., the strain localizes in a point. In the case of standard nonlocality, $m=1$, the first term in Eqs. 35 and 36 is always non-zero for each value of $k$. This implies that the waves are non-dissipative: the shape of an arbitrary loading wave cannot change to a stationary wave representing the localization band. For $m \leq 1$, the critical wavelength in Eq. 38 . which measures the localization band, is not defined and, consequently, the strain localizes into a point, like in a local formulation. On the other hand, for $m>1$ there exist some values of $k$, given by Eq. 37, which satisfy Eqs. 35 and 36 implying a finite dimension for the localization zone given by Eq. 38 .

Consider now the plasticity model in Eq. 32 with a nonlocal explicit-enhanced, in which the yield stress, Eq. 10, depends on the cumulative plastic strain and its second derivative, Eq. 23. Eqs. 33 and 34, together with Eq. 23, give the following equation system:

$$
E\left(\dot{u}_{, x x}-\dot{\kappa}_{, x}\right)=\rho \dot{u}_{, t t}
$$

$$
\dot{f}=E \dot{u}_{, x}-E \dot{\kappa}-H\left(\dot{\kappa}+\frac{\ell^{2}}{2} \dot{\kappa}_{, x x}\right)=0
$$

Let us analyze harmonic waves of frequency $\omega$ and wave number $k$, in which the velocity and cumulative plastic strain field are given by Eq. 15. Substituting Eq. 15 and its partial derivatives with respect to $x$ and $t$ into Eqs. 39 and 40, we obtain a linear system that must be satisfied at every point $x$ and every instant $t$. The homogeneous system has a non-trivial solution if and only if the determinant of the coefficient matrix is equal to zero:

$$
\begin{array}{r}
\left(E k^{2}-\rho \omega^{2}\right)\left[\left(1-\frac{\ell^{2}}{2} k^{2}\right) H+E\right] \\
-E^{2} k^{2}=0
\end{array}
$$

In statics $(\omega=0)$, we have

$$
\left(1-\frac{\ell^{2}}{2} k^{2}\right) H E k^{2}=0
$$

The perturbation does not remain homogeneous about the initial state if Eq. 42 is satisfied. The non-zero value of $k=k_{c r}$ which satisfies Eq. 42 is

$$
k_{\mathrm{cr}}=\frac{\sqrt{2}}{\ell}
$$

The corresponding critical wavelength is

$$
\lambda_{\mathrm{cr}}=\sqrt{2} \pi \ell
$$

For $\ell \rightarrow 0$ (i.e., for a local strain-softening model), the critical wavelength $\lambda_{c r}$ tends to zero, which means that the strain localizes into a point. For $\ell>0$, the critical wavelength has always a finite real value. Therefore, we conclude that the explicit gradient-type model is able to regularize the problem of softening plasticity. 
Consider now the nonlocal plasticity model in Eq. 32 with an implicit gradient-type enhancement. Two differentiations of Eq. 34 with respect to $x$, together with Eq. 28, give

$$
\dot{\hat{\kappa}}=\dot{\kappa}+\frac{E \ell^{2}}{2 H}\left(\dot{u}_{, x x x}-\dot{\kappa}_{, x x}\right)
$$

Rearranging Eqs. 33, 34 and 45, we obtain the following system of equations

$$
\begin{gathered}
E\left(\dot{u}_{, x x}-\dot{\kappa}_{, x}\right)=\rho \dot{u}_{, t t} \\
\dot{f}=E \dot{u}_{, x}-E \dot{\kappa}- \\
H\left(\dot{\kappa}+\frac{E \ell^{2}}{2 H}\left(\dot{u}_{, x x x}-\dot{\kappa}_{, x x}\right)=0\right.
\end{gathered}
$$

Consider now harmonic wave solutions of frequency $\omega$ and wave number $k$, with velocity and cumulative plastic strain field given by Eq. 15 . Substituting Eq. 15 and its partial derivatives with respect to $x$ and $t$ into Eqs. 46 and 47, one gets a system of two homogeneous linear equations, whose non-trivial solution exists if and only if the determinant of the coefficient matrix vanishes, i.e.

$$
\begin{array}{r}
\left(E k^{2}-\rho \omega^{2}\right)\left(E+H+E \frac{\ell^{2}}{2} k^{2}\right)- \\
E^{2} k^{2}\left(1+\frac{\ell^{2}}{2} k^{2}\right)=0
\end{array}
$$

For $\omega=0$ we have

$$
E H k^{2}=0
$$

This equation implies that the phase velocity $c_{p}=\omega / k$ is not a function of the wave number. So, the waves are non-dissipative. and the implicit gradient-type enhancement is unable to restore the well-posedness of the problem when softening occurs. This means that the implicit gradient formulation of the nonlocal plasticity model in Eq. 32 exhibits the same behavior as the nonlocal integral-type with $m=1$ (see Eq. 36).

\subsection{Ductile damage plasticity with nonlocal plastic variable}

In this section a plasticity model motivated by the concept of ductile damage proposed by
Geers, Engelen and coworkers [15, 16] will be considered in an over-nonlocal integral-type version. This model is characterized by the softening-hardening law written in the multiplicative format, in which the yield function in one-dimensional case is given by

$$
f(\sigma, \kappa, \hat{\kappa})=|\sigma|-\left[1-\omega_{p}(\hat{\kappa})\right]\left(\sigma_{0}+h \kappa\right)
$$

where the plastic modulus $h$ is a positive constant. In the yield function of Eq. 50 the current yield stress is defined by two terms: the term $\sigma_{0}+h \kappa$ which represent the linear plastic hardening of the bulk material and the term $1-\omega_{p}$ which reduces the yield stress for the growing of the damage in the material. An important feature is that the hardening is governing by the local cumulative plastic strain, the softening is driven by the nonlocal cumulative plastic strain. The essential component of the ductile damage model is the hardening-softening law written in the multiplicative form (Eq. 50), which differs from the hardening-softening law of Eq. 10 written in an additive format. A linear dependence of $\omega_{p}$ on $\hat{\kappa}$ is given by a piecewise linear law

$$
\omega_{p}(\hat{\kappa})= \begin{cases}0 & \text { if } \hat{\kappa} \leq \kappa_{i} \\ \frac{\hat{\kappa}-\kappa_{i}}{\kappa_{f}-\kappa_{i}} & \text { if } \kappa_{i} \leq \hat{\kappa} \leq \kappa_{f} \\ 1 & \text { if } \hat{\kappa} \geq \kappa_{f}\end{cases}
$$

where $\kappa_{i}$ is a material parameter which characterized the damage threshold and $\kappa_{f}$ is a material parameter which specifies the plastic deformation at failure (i.e. at zero yield stress). For this kind of over-nonlocal ductile damage model the Eqs 33 and 34 are written as

$$
\begin{aligned}
& E\left(\dot{u}_{, x x}-\dot{\kappa}_{, x}\right)=\rho \dot{u}_{, t t} \\
& \dot{f}=E \dot{u}_{, x}-E \dot{\kappa}- \\
& \left(1-\omega_{p}\right) h \dot{\kappa}+\left(\sigma_{0}+h \kappa_{0}\right) \dot{\omega}_{p}=0
\end{aligned}
$$

Localization takes place only in the softening range, in which, for $\kappa_{i} \leq \hat{\kappa} \leq \kappa_{f}$, we have

$$
\omega_{p}=\frac{\kappa_{0}-\kappa_{i}}{\kappa_{f}-\kappa_{i}} \quad \text { and } \quad \dot{\omega}_{p}=\frac{\dot{\hat{\kappa}}}{\kappa_{f}-\kappa_{i}}
$$

Using Eqs. 52, 53 and 54 and starting from an uniform initial state $\left(\epsilon(x)=\epsilon_{0}=\right.$ const and 
$\hat{\kappa}(x)=\kappa(x)=\kappa_{0}=$ const) one obtains the system of two equations which characterized the localization problem. Setting the determinant of the coefficient matrix of this system equal to zero one gets

$$
\begin{aligned}
& {\left[-\frac{\kappa_{f}-\kappa_{0}}{\kappa_{f}-\kappa_{i}} h+\frac{\sigma_{0}-h \kappa_{0}}{\kappa_{f}-\kappa_{i}}(1-m+\right.} \\
& \left.\left.\quad \frac{m}{\ell} A(k)\right)\right]\left[E k^{2}-\rho \omega^{2}\right]+E \rho \omega^{2}=0
\end{aligned}
$$

In statics $(\omega \rightarrow 0)$, Eq. 55 becomes

$$
\begin{aligned}
& {\left[-\frac{\kappa_{f}-\kappa_{0}}{\kappa_{f}-\kappa_{i}} h+\frac{\sigma_{0}+h \kappa_{0}}{\kappa_{f}-\kappa_{i}}\right.} \\
& \left.\quad\left(1-m+\frac{m}{\ell} A(k)\right)\right] E k^{2}=0
\end{aligned}
$$

As done in the foregoing for other plasticity models, first we substitute the value of $A(k)=$ $\ell e^{-k^{2} \ell^{2} / 4 \pi}$ into Eq. 56, and then we search for the value of the critical wave number $(k \neq 0)$ for which Eq. 56 can be satisfied

$$
\sqrt{\ln \left(\frac{k_{\mathrm{cr}}=\frac{2 \sqrt{\pi}}{\ell}}{h\left(\kappa_{f}-\kappa_{0}\right) /\left(\sigma_{0}+h \kappa_{0}\right)+m-1}\right)}
$$

The corresponding critical wavelength is

$$
\begin{gathered}
\lambda_{\mathrm{cr}}=2 \pi / k_{\mathrm{cr}}=\ell \sqrt{\pi} \\
{\left[\ln \left(\frac{m}{h\left(\kappa_{f}-\kappa_{0}\right) /\left(\sigma_{0}+h \kappa_{0}\right)+m-1}\right)\right]^{-\frac{1}{2}}}
\end{gathered}
$$

Note that for $m \rightarrow 0$, or for $\ell \rightarrow 0$ (local strain-softening model), the critical wavelength $\lambda_{c r}$ tends to zero, i.e., the strain localizes in a point. For each value of the parameter $m$, in particular for $m=1$ (standard nonlocality), the critical wavelength, which measures the localization band, is well defined and, consequently, the strain localizes into a band with a finite dimension. Another important feature of this nonlocal plasticity model is that the critical wavelength in Eq. 58 is a decreasing function of the softening parameter $\kappa_{0}$. Thus, the width of the localization band tends to reduce to zero as the softening parameter increases $\left(\lambda_{\mathrm{cr}} \rightarrow 0\right.$ for $\kappa_{0} \rightarrow \kappa_{f}$ and $m=1$ ), i.e. there is convergence to a discrete crack. Note that this important feature happens only for the standard nonlocality ( $m=1)$. We may conclude that the ductile damage model proposed by Geers, Engelen and coworkers with the integral-type nonlocal version provides a full regularization of the softening problem even for the standard nonlocal formulation with $m=1$.

Consider now the ductile damage model, in which the yield stress, Eq. 50, now depends on the cumulative plastic strain and its second derivative as expressed by Eq. 23. For the linear softening in Eq. 51, the equation system that governs the localization problem of the ductile damage model with explicit gradient enhancement is given by

$$
\begin{gathered}
E\left(\dot{u}_{, x x}-\dot{\kappa}_{, x}\right)=\rho \dot{u}_{, t t} \\
\dot{f}=E \dot{u}_{, x}-E \dot{\kappa}-\frac{\kappa_{f}-\kappa_{0}}{\kappa_{f}-\kappa_{i}} h \dot{\kappa}+ \\
\frac{\sigma_{0}+h \kappa_{0}}{\kappa_{f}-\kappa_{i}}\left(\dot{\kappa}+\frac{\ell^{2}}{2} \dot{\kappa}_{, x x}\right)=0
\end{gathered}
$$

If the harmonic waves of frequency $\omega$ and wave number $k$, propagating along the bar, Eq. 15 are substituted in Eqs. 59 and 60, one obtains an homogeneous system of two linear equations that has a non-trivial solution if and only if the determinant of the coefficient matrix vanishes:

$$
\begin{aligned}
& \left(E k^{2}-\rho \omega^{2}\right)\left[-E-\frac{\kappa_{f}-\kappa_{0}}{\kappa_{f}-\kappa_{i}} h+\right. \\
& \left.\quad \frac{\sigma_{0}+h \kappa_{0}}{\kappa_{f}-\kappa_{i}}\left(1-\frac{\ell^{2}}{2} k^{2}\right)\right]+E^{2} k^{2}=0
\end{aligned}
$$

In statics $(\omega=0)$, we have

$$
\begin{array}{r}
{\left[-\frac{\kappa_{f}-\kappa_{0}}{\kappa_{f}-\kappa_{i}} h+\frac{\sigma_{0}+h \kappa_{0}}{\kappa_{f}-\kappa_{i}}\left(1-\frac{\ell^{2}}{2} k^{2}\right)\right]} \\
E k^{2}=0(62)
\end{array}
$$

The non-zero value of $k$ that satisfies Eq. 62 is

$$
k_{\mathrm{cr}}=\frac{\sqrt{2}}{\ell}\left(1-\frac{\kappa_{f}-\kappa_{0}}{\sigma_{0}+h \kappa_{0}} h\right)^{1 / 2}
$$

The corresponding critical wavelength is

$$
\lambda_{\mathrm{cr}}=\sqrt{2} \pi \ell\left(1-\frac{\kappa_{f}-\kappa_{0}}{\sigma_{0}+h \kappa_{0}} h\right)^{-1 / 2}
$$


Since, for $\ell>0$, the critical wavelength always has a finite real value, the explicit gradient enhancement of the ductile damage model is able to regularize the problem of softening plasticity. Note that the explicit gradient enhancement of the ductile damage model provides a critical wavelength which does not tend to zero at complete failure $\left(\kappa_{0} \rightarrow \kappa_{f}\right)$.

Consider now the ductile damage model with an implicit gradient enhancement. Two differentiations of Eq. 34 with respect to $x$, together with Eq. 28, furnish

$$
\begin{aligned}
\dot{\hat{\kappa}}=\dot{\kappa}+ & \frac{\ell^{2}}{2}\left(-E \dot{u}_{, x x x}+E \dot{\kappa}_{, x x}+\right. \\
& \left.\frac{\kappa_{f}-\kappa_{0}}{\kappa_{f}-\kappa_{i}} h \dot{\kappa}_{, x x}\right) \frac{\kappa_{f}-\kappa_{i}}{\sigma_{0}+h \kappa_{0}}
\end{aligned}
$$

Using the linear softening given by Eq. 51, the equation system that governs the localization problem now has the form:

$$
\begin{gathered}
E\left(\dot{u}_{, x x}-\dot{\kappa}_{, x}\right)=\rho \dot{u}_{, t t} \\
\dot{f}=E \dot{u}_{, x}-E \dot{\kappa}-\frac{\kappa_{f}-\kappa_{0}}{\kappa_{f}-\kappa_{i}} h \dot{\kappa}+\frac{\sigma_{0}+h \kappa_{0}}{\kappa_{f}-\kappa_{i}} \dot{\kappa} \\
+\frac{\ell^{2}}{2}\left(E \dot{\kappa}_{, x x}-E \dot{u}_{, x x x}+\frac{\kappa_{f}-\kappa_{0}}{\kappa_{f}-\kappa_{i}} h \dot{\kappa}_{, x x}\right)=0(67)
\end{gathered}
$$

If harmonic waves of frequency $\omega$ and wave number $k$, Eq. 15, are substituted in Eqs. 66 and 67, one gets a homogeneous system of two linear equations that has a non-trivial solution if and only if the determinant of the coefficient matrix is equal to zero:

$$
\begin{gathered}
\left(E k^{2}-\rho \omega^{2}\right)\left[-E\left(1+\frac{\ell^{2}}{2} k^{2}\right)-\right. \\
\left.\frac{\kappa_{f}-\kappa_{0}}{\kappa_{f}-\kappa_{i}} h\left(1+\frac{\ell^{2}}{2} k^{2}\right)+\frac{\sigma_{0}+h \kappa_{0}}{\kappa_{f}-\kappa_{i}}\right]+ \\
E^{2} k^{2}\left(1+\frac{\ell^{2}}{2} k^{2}\right)=0
\end{gathered}
$$

In statics $(\omega=0)$, we have

$$
\begin{aligned}
& {\left[-\frac{\kappa_{f}-\kappa_{0}}{\kappa_{f}-\kappa_{i}} h\left(1+\frac{\ell^{2}}{2} k^{2}\right)+\right.} \\
&\left.\frac{\sigma_{0}+h \kappa_{0}}{\kappa_{f}-\kappa_{i}}\right] E k^{2}=0
\end{aligned}
$$

The non-zero value of $k=k_{c r}$ that satisfies Eq. 69 is

$$
k_{\mathrm{cr}}=\frac{\sqrt{2}}{\ell}\left(\frac{\sigma_{0}+h \kappa_{0}}{\left(\kappa_{f}-\kappa_{0}\right) h}-1\right)^{1 / 2}
$$

The corresponding critical wavelength is

$$
\lambda_{\mathrm{cr}}=\sqrt{2} \pi \ell\left(\frac{\sigma_{0}+h \kappa_{0}}{\left(\kappa_{f}-\kappa_{0}\right) h}-1\right)^{-1 / 2}
$$

Since $\ell>0$, the critical wavelength, given by Eq. 71, always has a finite real value. This implies that the implicit gradient enhancement of the ductile damage model achieves regularization of the problem due to the softening. Moreover, the two critical wavelength Eqs. 58 and 71, which differ from that obtained for the explicit gradient enhancement, Eq. 64, ensure convergence to zero upon complete failure $\left(\kappa_{0} \rightarrow \kappa_{f}\right)$.

\section{LOCALIZATION ANALYSIS OF NON- LOCAL DAMAGE MODEL}

Following the approach of [25], let us now analyze the localization of an over-nonlocal version of the scalar continuous damage model proposed in [7]. The constitutive relation in one-dimension and the loading function are

$$
\begin{gathered}
\sigma=(1-d) E \epsilon \\
f(\hat{Y}, d)=\int_{0}^{\hat{Y}} F(z) \mathrm{d} z-d
\end{gathered}
$$

where $d$ is the damage variable, function $F$ defined the damage evolution, and $\hat{Y}$ is the overnonlocal average of the energy release rate:

$$
\begin{array}{r}
\hat{Y}(x)=\int_{V} \alpha_{m}^{*}(\xi-x) Y(\xi) \mathrm{d} \xi \\
Y(x)=\frac{1}{2} E \epsilon(x)^{2}
\end{array}
$$

In the case of associated damage, the evolution of the damage variable is controlled by the conditions:

$$
\dot{d} \geq 0 \quad f \leq 0 \quad \dot{d} f=0
$$

Using the same approach as in the previous section (Eqs. 13 14 ) and assuming an initial state of uniform damage and uniform strain 
field, i.e. $\hat{\epsilon}(x)=\epsilon(x)=\epsilon_{0}=$ const. and $d(x)=d_{0}=$ const., after some mathematical manipulations and substitution of the the previous equations, one obtain the equation of motion. Considering a harmonic wave perturbation of frequency $\omega$ and wave number $k$ propagating through an infinite bar (Eq. 15) the equation of motion has a non-zero (non-trivial) solution only if the following term vanishes

$$
\begin{gathered}
\omega^{2}=k^{2} \frac{E}{\rho}\left[1-d_{0}-\right. \\
\left.E \epsilon_{0}^{2} F(\hat{Y})\left(1-m+\frac{m}{\ell} A(k)\right)\right]
\end{gathered}
$$

Considering the same constitutive law and the same parameters as [25]

$$
F(\hat{Y})=\frac{b_{1}+2 b_{2}\left(\hat{Y}-Y^{0}\right)}{\left[1+b_{1}\left(\hat{Y}-Y^{0}\right)+b_{2}\left(\hat{Y}-Y^{0}\right)^{2}\right]^{2}}
$$

Because we assumed an infinite body and a uniform initial state, we have $\hat{Y}=Y=\frac{1}{2} E \epsilon_{0}^{2}$ and $d_{0}=\int_{0}^{\hat{Y}} F(z) \mathrm{d} z$. Using $A(k)=\ell e^{-k^{2} \ell^{2} / 4 \pi}$, the critical wave number $k_{\mathrm{cr}}$ that makes the phase velocity and the angular frequency vanish $\left(c_{p}=\omega=0\right)$ is

$$
\begin{gathered}
k_{\mathrm{cr}}=\frac{2 \sqrt{\pi}}{\ell} \\
{\left[\ln \left(\frac{m E \epsilon_{0}^{2} F(\hat{Y})}{1-d_{0}+(m-1) E \epsilon_{0}^{2} F(\hat{Y})}\right)\right]^{1 / 2}}
\end{gathered}
$$

and the corresponding critical wavelength is:

$$
\begin{gathered}
\lambda_{\mathrm{cr}}=2 \pi / k_{\mathrm{cr}}=\ell \sqrt{\pi} \\
{\left[\ln \left(\frac{m E \epsilon_{0}^{2} F(\hat{Y})}{1-d_{0}+(m-1) E \epsilon_{0}^{2} F(\hat{Y})}\right)\right]^{-1 / 2}}
\end{gathered}
$$

Because, in the case of standard nonlocality $(m=1)$, the critical wavelength is well defined, the nonlocal damage model does not necessitate an over-nonlocal formulation. The standard nonlocality suffices to correct the problems of local softening for a damage model with the average of the damage energy release rate. Moreover, the critical wavelength of Eq. 78 goes to zero upon complete failure which means that the localization band converges to a discrete crack.

Consider the continuous damage model in Eq. 72 that is now regularized by an explicit gradient-type formulation. The rate of the nonlocal energy release rate is given by

$$
\dot{\hat{Y}}(x)=\dot{Y}(x)+\frac{\ell^{2}}{2} \dot{Y}(x)_{, x x}
$$

Using the definition of the rate of the energy release rate and following the previous mathematical derivation one obtains the critical wave number, $k_{\mathrm{cr}}$, which makes the phase velocity and the angular frequency vanish $\left(c_{p}=\omega=0\right)$,

$$
k_{\mathrm{cr}}=\frac{\sqrt{2}}{\ell} \sqrt{\frac{d_{0}-1+E \epsilon_{0}^{2} F(\hat{Y})}{E \epsilon_{0}^{2} F(\hat{Y})}}
$$

and the corresponding critical wavelength is:

$$
\lambda_{\text {cr }}=2 \pi / k_{\text {cr }}=\pi \sqrt{2} \ell \sqrt{\frac{E \epsilon_{0}^{2} F(\hat{Y})}{d_{0}-1+E \epsilon_{0}^{2} F(\hat{Y})}}
$$

The behavior of the explicit gradient-type damage model differs from the nonlocal damage model in terms of the width of the localization band. For the complete loss of material integrity, $d=1$, the explicit gradient-type model provides a finite width of the localization band equal to $\pi \sqrt{2} \ell$.

For the same damage model consider an implicit gradient-type model, in which the rate of the nonlocal energy release rate is the solution of the following Helmholtz partial differential equation

$$
\dot{\hat{Y}}(x)-\frac{\ell^{2}}{2} \dot{\hat{Y}}(x)_{, x x}=\dot{Y}(x)
$$

Using the same previous procedure one obtains the critical wave number as

$$
k_{\mathrm{cr}}=\frac{\sqrt{2}}{\ell} \sqrt{\frac{d_{0}-1+E \epsilon_{0}^{2} F(\hat{Y})}{1-d_{0}}}
$$

and the corresponding critical wavelength is:

$$
\lambda_{\mathrm{cr}}=2 \pi / k_{\mathrm{cr}}=\pi \sqrt{2} \ell \sqrt{\frac{1-d_{0}}{d_{0}-1+E \epsilon_{0}^{2} F(\hat{Y})}}
$$


We observe that the behaviors of the implicittype gradient model and the nonlocal damage model (dashed line) are similar: both models predict a narrowing localization band, the width of which tends to zero for $d \rightarrow 1$. This is not surprising because the implicit model exhibits strong nonlocality.

\section{CONCLUSIONS}

The localization analyses performed reveal that the effectiveness of the regularization achieved by integral-type nonlocality depends on the particular type of nonlocal plasticity model adopted. For the nonlocal plasticity model, in which the plastic strain is replaced by its nonlocal average in the stress-strain law, the harmonic waves are non-dissipative and the constitutive model is unable to reproduce the localization band due to the softening. The nonlocal plasticity model, in which the yield stress depends on the nonlocal cumulative plastic strain, provides correct regularization only if the over-nonlocal formulation is adopted with $m$ greater than 1 . The nonlocal integral-type formulation of the ductile damage model [15] is able to give a correct localization behavior even for the standard nonlocality with $m=1$ (i.e. the critical wavelength, has positive real value also for $m=1$ ). When a nonlocal damage model is considered, the over-nonlocal approach is not needed (i.e. $m=1$ ).

The localization analysis of the nonlocal integral-type models also shows that a narrowing of the critical wavelength is obtained only for the nonlocal generalizations of the ductile damage model and the damage model.

The localization analysis of the implicit gradient-type model based on the Helmoltz equation reveals that this formulation is able to regularize the softening behavior of the damage model and the ductile damage model, but not the nonlocal plasticity model.

The localization analysis of the gradient-type models shows that a narrowing of the localization band is obtained only for the implicit gradient enhancement of the ductile damage model and the damage model. By contrast, the explicit gradient enhancement of all the considered softening models gives a critical wavelength that does not tend to zero at complete failure.

\section{REFERENCES}

[1] Bažant, Z.P., Belytschko, T., 1985. Wave propagation in strain-softening bar: exact solution. Journal of Structural Engineering, ASCE 111:381-389.

[2] Bažant, Z.P., Oh, B.H., 1983. Crack band theory for fracture of concrete. Material and Structures, RILEM 16(93):155-177.

[3] Lasry, D., Belytschko, T., 1988. Localization limiter in transient problem. International Journal of Solids and Structure 24:581-597.

[4] Eringen, A.C., 1966. A unified theory of thermomechanical materials. International Journal of Engineering Science 4:179-202.

[5] Kröner, E., 1967. Elasticity theory of materials with long-range cohesive force. International Journal of Solids and Structures 3:731-742.

[6] Bažant, Z.P., Chang, T.P., 1984. Instability of nonlocal continuum and strain averaging. Journal of Engineering Mechanics, ASCE 110:1441-1450.

[7] Pijaudier-Cabot, G., Bažant, Z.P., 1987. Nonlocal damage theory. Journal of Engineering Mechanics, ASCE 113:15121533.

[8] Vermeer, P.A., Brinkgreve, R.B.J., 1994. A new effective non-local strain measure for softening plasticity. In R. Chambon, J. Desrues, I. Vardoulakis (Eds.), Localization and Bifurcation Theory for Soil and Rocks; Balkema, Rotterdam, pp. 89-100.

[9] Aifantis, E.C. 1984. On the microstructural origin of certain inelastic models. Journal of Engineering Matematical Technology 106:326-330. 
[10] Zbib, H.M., Aifantis, E.C., 1988. On the localization and postlocalization behaviour of plastic deformation, I, II, III. Res Mechanica 23:261-277, 279-292, 293-305.

[11] Mühlhaus, H.B., Aifantis, E.C., 1991. A variational principle for gradient plasticity. International Journal of Solids ans Structure 28:845-858.

[12] Pamin, J., 1994. Gradient-dependent plasticity in numerical simulation of localization phenomena, $\mathrm{PhD}$ Dissertation, TU Delft, Netherland.

[13] Peerlings, R.H.J., de Borst, R., Brekelmans, W.A.M., de Vree, J.H.P., Spee, I. 1996. Some observations on localisation in non-local and gradient damage models. European Journal of Mechanics A/Solids 15(6):937-953.

[14] Di Luzio, G., and Bažant, Z.P. 2005. Spectral analysis of localization in nonlocal and overnonlocal materials with softening plasticity or damage. International Journal of Solids and Structures 42(23):60716100.

[15] Geers, M.G.D., Engelen, R.A.B., Ubachs, R.L.J.M., 2001. On the numerical modelling of ductile damage with an implicit gradient-enahced formulation. Revue Europeenne des Elements Finis 10(23-4):173-192.

[16] Engelen, R.A.B., Geers, M.G.D., Baaijens, F.P.T., 2003. Nonlocal implicit gradient-enhanced elasto-plasticity for modelling of softening behaviour. International Journal of Plasticity 19:403-433.

[17] Sluys, L.J., 1992. Wave propagation, localization and dispersion in softening solids, PhD Dissertation, TU Delft, Netherland.

[18] de Borst, R., Pamin, J., Peerlings, R. H. J., Sluys, L. J. 1995. On gradient-enhanced damage and plasticity models for failure in quasi-brittle and frictional materials. Computational Mechanincs 17(1-1):130141.

[19] Peerlings, R.H.J., de Borst, R., Brekelmans, W.A.M.,de Vree, J.H.P., 1996. Gradient-enhanced damage for quasibrittle materials. International Journal for Numerical Methods in Engineering 39:3391-3403.

[20] Peerlings, R.H.J., Geers, M.G.D., de Borst, R. and Brekelmans, W.A.M. 2001. A critical comparison of nonlocal and gradient-enhanced softening continua. International Journal of Solids and Structure 38:7723-7746.

[21] Peerlings, R.H.J., de Borst, R. and Brekelmans, W.A.M., Geers, M.G.D., 2002. Localization issue in local and nonlocal continuum approaches to fracture. European Journal of Mechanics A/Solids 21:175189.

[22] Comi, C., Rizzi, E., 2000. On bifurcation in local and nonlocal materials with tension and comprension damage. Proceedings of ECCOMAS 2000, Barcellona, Spain.

[23] Bažant, Z.P., Jirásek, M. 2002. Nonlocal Integral Formulation for Plasticity and Damage: Survey of Progress. Journal of Engineering Mechanics, ASCE 128(11):1119-1149.

[24] Jirásek, M., Rolsoven, S., 2003. Comparison of integral-type nonlocal plasticity models for strain-softening materials. International Journal of Engineering Science 41:1553-1602.

[25] Pijaudier-Cabot, G., Benallal, A., 1993. Strain localization and bifurcation in a nonlocal continuum. International Journal of Solids and Structure 30:1761-1775. 